\title{
Design and specification of analog artificial neural network
}

\author{
Hassan Jouni ${ }^{1,2} \cdot$ Adnan Harb $^{3}$ (I) $\cdot$ Gilles Jacquemod $^{1} \cdot$ Yves Leduc $^{1}$
}

Received: 25 January 2019 / Accepted: 6 September 2019 / Published online: 29 October 2019

(c) Springer Nature Switzerland AG 2019

\begin{abstract}
In this paper, we have implemented, using Matlab Simulink an analog artificial neural network for breast cancer classification. Simulated results with ideal building blocks exhibit a total error of classification of $2.6 \%$. Thanks to this value, we have modified Simulink models of the building blocks (i.e. multiplier, activation function and its derivative) in order to take into account their non-idealities. This study allows to determine their influence on the classification quality and to extract some specifications of these building blocks.
\end{abstract}

Keywords Neural network - MLP · Breast cancer - Non-ideal blocks · Simulink

\section{Introduction}

Artificial Neural Networks (ANN) are used in many fields such as medical domain (the current paper application), communication, power control and other applications.

ANN have two methods of implementation:

1. Implementation by using software.

2. Implementation by using hardware.

The first method uses software like $C++$, MATLAB, LabVIEW and other software using different algorithm like Multilayer perceptron with Back-propagation (MLP), Radial Basis Function (RBF), Support Vector Machine (SVM) [1-3]. Some published papers use the Fuzzy logic method [4] and others use the normalized Multilayer perceptron with Back-propagation (MLP) [5].

In the second method, we have two options:

1. Digital hardware implementation.

2. Analog hardware implementation.

In the first one, digital devices are used for example the Field Programmable Gate Array (FPGA) [6-8] and [9].
The second way is based on analog implementation of ANN [10], this type of artificial neural networks is particularly interesting for CMOS VLSI implementations because every parallel element (neuron or synapse) is relatively simple, allowing the complete integration of big networks on a single chip [11]. Multipliers [12], nonlinear function and its derivative $[13,14]$ are essential key elements in the analog signal processing in particular for analog VLSI implementation of artificial neural networks. In this article, we create a design for breast cancer detection and classification (i.e. malignant or benign) by using the artificial neural network MLP (Multi-Layer Perceptron) with back-propagation [14-16], based on the Wisconsin Data Base [17]. In a previous work, we have demonstrated that the most efficient architecture consists of 9 inputs neurons corresponding to 9 attributes of the Data Base, 10 neurons in the hidden layer and 2 for the output layer (two output classes: Benign or malignant), named 9-10-2 without biases [18]. The main objective of this work is to determine the influence of the nonidealities of the analog building blocks on the quality of the classification and to determine the specifications of each building block. Thanks to the HCMOS9A $130 \mathrm{~nm}$

\footnotetext{
$\triangle$ Adnan Harb, adnan.harb@b-iu.edu.lb|'Polytech'Lab, EA UNS 7498, Université Côte d'Azur, 930 Chemin des Colles, 06410 Biot, France. ${ }^{2}$ Department of Electrical and Electronics Engineering, Lebanese International University, Beirut, Lebanon. ${ }^{3}$ Department of Electrical and Electronics Engineering, International University of Beirut, Beirut, Lebanon.
} 
STMicroelectronics technology [12], we create non-ideal multiplier, activation function and its derivative blocks, under Simulink Matlab R2016a [19].

This paper is organized as follows. Section 2 highlights the Simulink implementation of the neural network. Ideal models of building blocks are created and simulated results are presented in Sect. 3. In Sect. 4, these models are modified in order to take into account the limitations of the CMOS analog implementation of these building blocks. Finally, conclusion and trends are given in Sect. 5 .

\section{Definition of cancer}

All blocks of body were created from cells [20]. In normal case cells grow and divide to form new cells and when cells grow out of control will be a cancer. If these cells grow inside the breast form a tumor [21], and will be benign (not cancer) or malignant (cancer). From different parts of the breast, the breast cancers can begin [21].

\section{Wisconsin breast cancer dataset}

The Wisconsin Breast Cancer database [22] is located in the UCI Machine Learning Repository and it is applied in this research.

Breast-cancer-Wisconsin dataset has:

- 699 instances (Benign: 458 Malignant: 241).

- 9 integer-valued attributes.

- 2 classes: 212 malignant and 357 benign, or $(65.5 \%$ malignant and $34.5 \%$ benign).

- Missing attribute values: none.

We can find also this database from the following link: ftp.cs.wisc.edu cd math-prog/cpo-dataset/machine-learn/ WDBC/.

\section{Dataset description [17]}

The Wisconsin Breast Cancer dataset [17] is applied, to classify malignant (cancerous) from benign (non-cancerous) samples. A brief specification of this dataset

Table 1 Specification of the breast cancer dataset

\begin{tabular}{llll}
\hline Dataset & No. of attributes & No. of instances & No. of classes \\
\hline $\begin{array}{l}\text { Wisconsin } \\
\text { breast cancer } \\
\text { (original) }\end{array}$ & 9 & 699 & 2 \\
\hline
\end{tabular}

is presented in Table 1 [17]. The dataset consists of some classification patterns with a set of numerical attributes.

\section{Data set information [17]}

Dr. Wolberg reports his clinical cases periodically and grouping this dataset by recorded date. The detail of this dataset is listed in the following Table 2. Table 3 represents the 9 attributes with small description and will be 9 inputs to the neural network.

Classification is one of the most significant and fundamental tasks in neural network. Wisconsin breast cancer medical datasets used to classify breast cancer and will be the output of the neural network:

1. Benign or 0 .

2. Malignant or 1.

Table 2 distribution of 699 instances

\begin{tabular}{lcl}
\hline Group & Instance & Date \\
\hline 1 & 367 & January 1989 \\
2 & 70 & October 1989 \\
3 & 31 & February 1990 \\
4 & 17 & April 1990 \\
5 & 48 & August 1990 \\
6 & 49 & Updated January 1991 \\
7 & 31 & June 1991 \\
8 & 86 & November 1991 \\
Total & 699 & As of the donated \\
& & database on 15 July \\
& & 1992 \\
\hline
\end{tabular}

Table 3 Wisconsin breast cancer dataset attributes

\begin{tabular}{lll}
\hline Id number & Attribute & Domain \\
\hline 1 & Clump thickness & $1-10$ \\
2 & Uniformity of cell size & $1-10$ \\
3 & Uniformity of cell shape & $1-10$ \\
4 & Marginal adhesion & $1-10$ \\
5 & Single epithelial cell size & $1-10$ \\
6 & Bare nuclei & $1-10$ \\
7 & Bland chromatin & $1-10$ \\
8 & Normal nucleoli & $1-10$ \\
9 & Mitoses & $1-10$ \\
\hline
\end{tabular}




\section{Simulink neural network implementation}

\subsection{Feed forward neural network}

The design of the Artificial Neural Network without Biases is presented in Fig. $1 \mathrm{a}$ and the equivalent scheme created in Simulink is shown in Fig. 1 b.

The equations, without biases, for the hidden and the output layers are given by Eqs. (1) and (2).

$a_{j}=g_{j}\left(\sum_{i=1}^{9} w_{i j} * x_{i}\right) \quad \mathrm{j}=1,2 \ldots 10$

$s_{k}=g_{k}\left(\sum_{j=1}^{10} w_{j k} * a_{j}\right) \quad k=1,2$

where $x_{i}$ are the inputs (the 9 Cancer attributes of the Wisconsin Data Base), $\mathrm{W}_{\mathrm{ij}}$ (or $\mathrm{wH}$ ) are the 90 coefficients of the hidden layer, $a_{j}$ are the 10 outputs of the hidden layer (i.e. the inputs of the output layer), $\mathrm{W}_{\mathrm{jk}}$ (or $\mathrm{wO}$ ) are the 20 coefficients of the output layer and $s_{k}$ are the 2 outputs of the neural network. $g_{j}$ is the activation function of the hidden layer and $g_{k}$ is the activation function of the output layer. A logsigmoid function was chosen as activation function for both hidden and output layers [18].

Due to the HCMOS9a technology and the power supply of the circuits $( \pm 0.9 \mathrm{~V}[12])$, we have chosen a dynamic of -0.9 to 0.9 for the coefficients (i.e. the weights, $\mathrm{W}_{\mathrm{ij}}$ and $\mathrm{W}_{\mathrm{jk}}$, of the neurons). Our reference for breast cancer detection and classification are located in neural network toolbox of Matlab R2016a [19]:

1. Number of inputs equal to 9 .

2. Number of outputs equal to $2=$ Number of neurons in output layer.

3. Number of neurons in hidden layer equal to 10 .

4. Number of samples equal to 699.

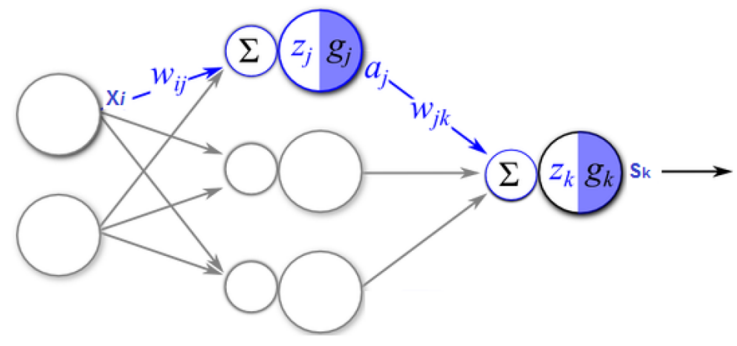

a Architecture of the Neural Network without Biases
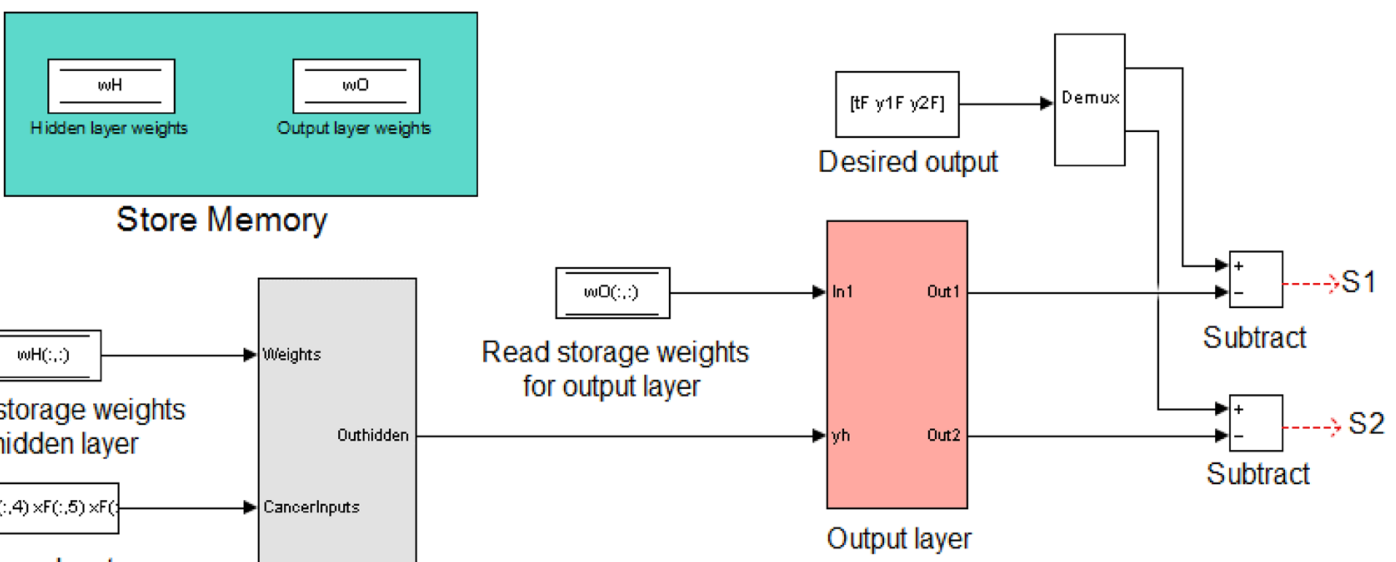

Hidden layer

b Feed-forward neural network

Fig. 1 a Architecture of the Neural Network without Biases, b feed-forward neural network 


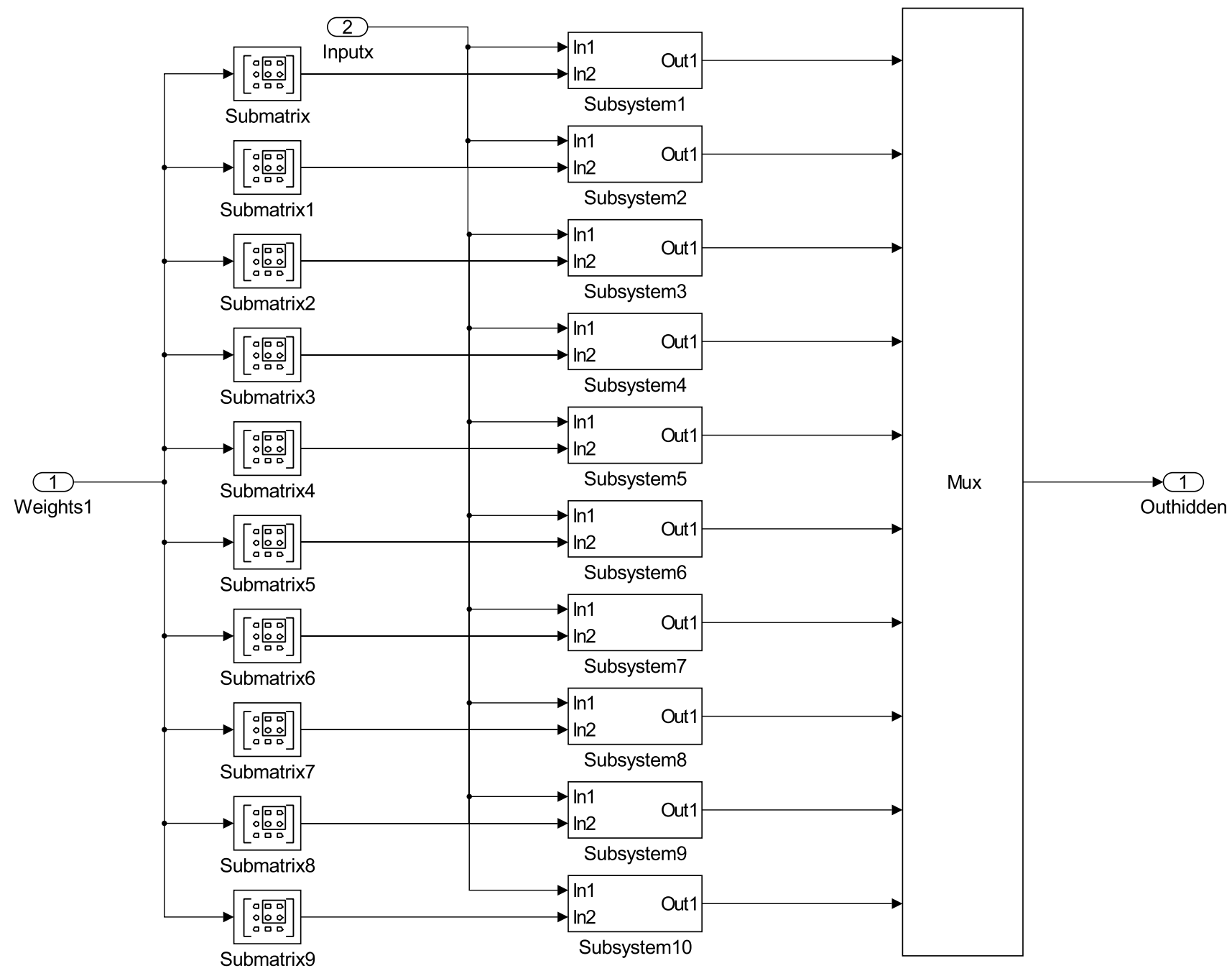

Fig. 2 Hidden layer details

5. Number of samples for learning process equal to 489 .

6. Number of epochs equal to 14 .

7. Learning rate equal to 0.25 .

\subsection{Memory package}

Before starting the learning process in our model, we must initialize the weights in hidden layer $\mathrm{wH}$ and the weights in output layer wO (cf. Fig. 1b) by applying the random function that creates a random value between -0.9 and 0.9 . This value will be stored in the memories package $\mathrm{wH}$ and $\mathrm{wO}$ and will be used in the future as inputs for analog CMOS multiplier.
The "Read storage weights for output layer" block is used to read the weight's values in output layer, and the "Read storage weights for hidden layer" block is used to read the weight's values in hidden layer.

\subsection{Hidden oud output layers}

The CancerTargets, the target output and the connection between hidden and output layers, are realized with Simulink routing [23]. We can find the Simulink method to build the artificial neural network in several papers for example the radial basis function RBF [24] or self-organizing map SOM artificial network [25]. 


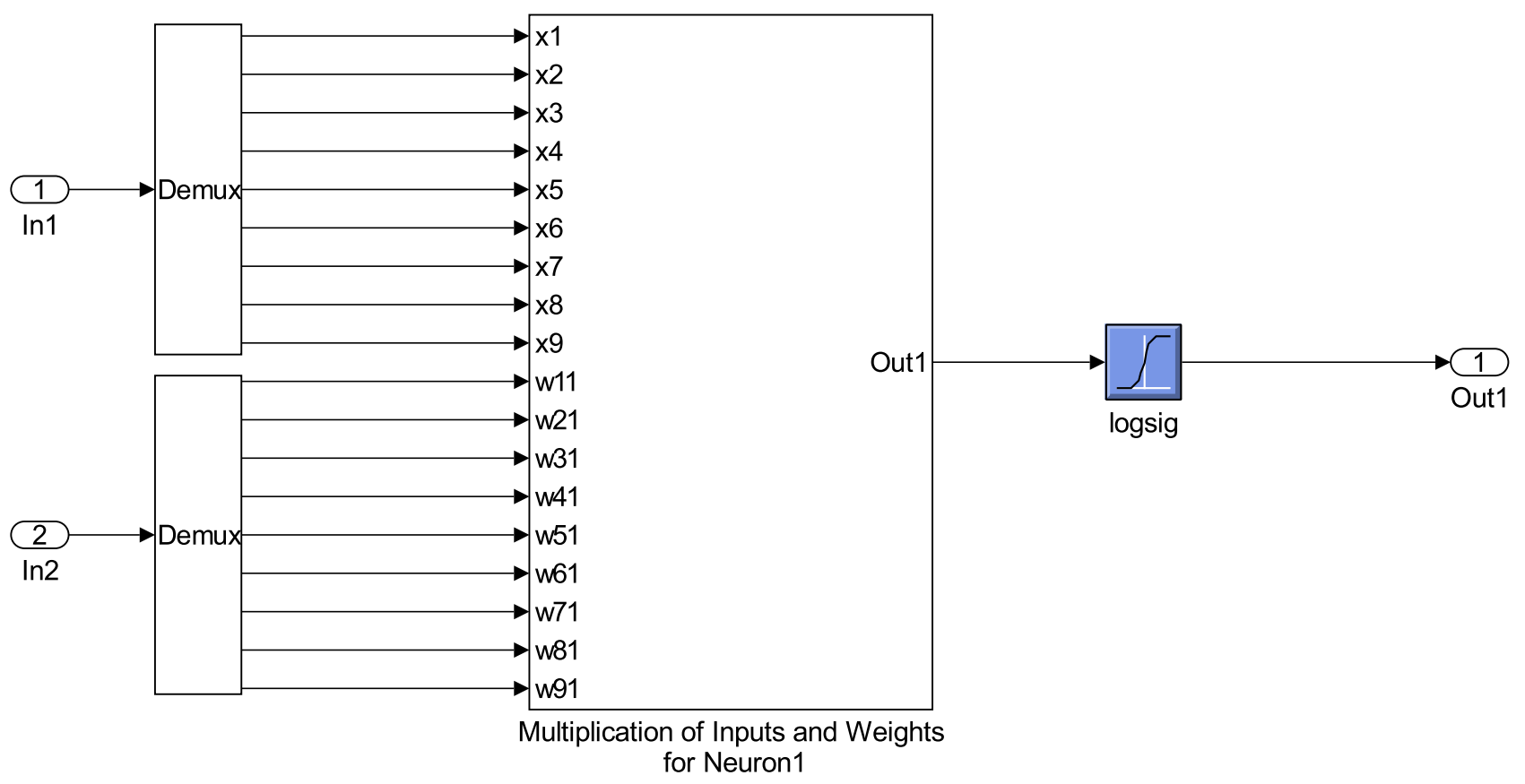

Fig. 3 Single neuron

The details of "Hidden Layer" block in Fig. 1 are presented in Fig. 2.

We choose through the submatrix block the corresponding weights for each neuron and we find in Fig. 3 the details for each neuron block in Fig. 2 and equivalent to the equation:

$a_{j}=\operatorname{Logsig}\left(\sum_{i=1}^{9} w_{i j} * x_{i}\right) \quad \mathrm{j}=1,2 \ldots 10$

The same for the output layer in Fig. 1b, the "Desired Output" block represents the target category, which is considered benign in element 1 and malignant in element 2 [23]. The "Cancerlnputs" block represents the nine attributes of 699 biopsies.

To separate the desired output 1 and desired output 2, the Demux block is used, and the "Subtract" block is used to generate the subtraction between the target outputs ( 1 or 2 ) and the neuron output ( 1 or 2 ).

Figure 4 shows the details of multiplication of inputs and weights values equivalent to the Eq. (4).

$$
\sum_{i=1}^{9} w_{i j} * x_{i} \quad \mathrm{j}=1,2 \ldots 10
$$

\subsection{Back-propagation}

To obtain the full design, depicted in Fig. 5, we add the backpropagation blocks and the feed-forward blocks.

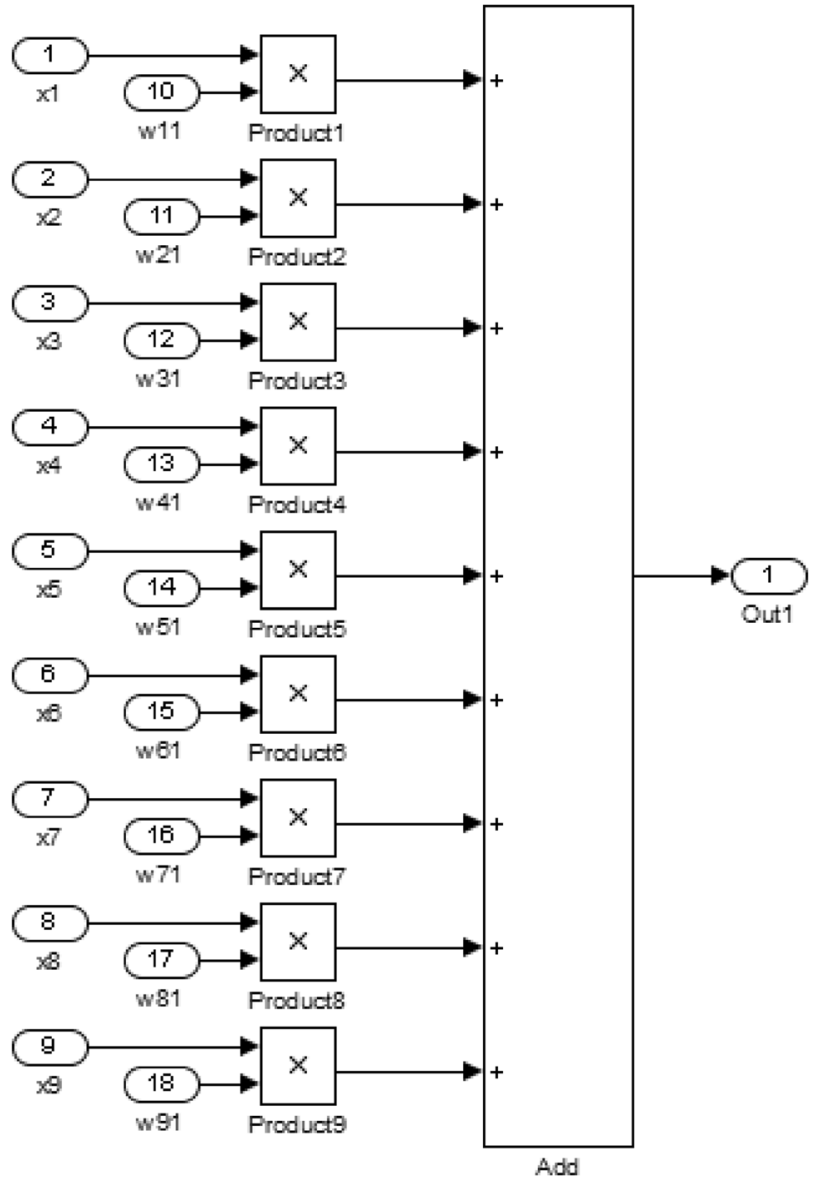

Fig. 4 Cancer inputs and weights multiplication 


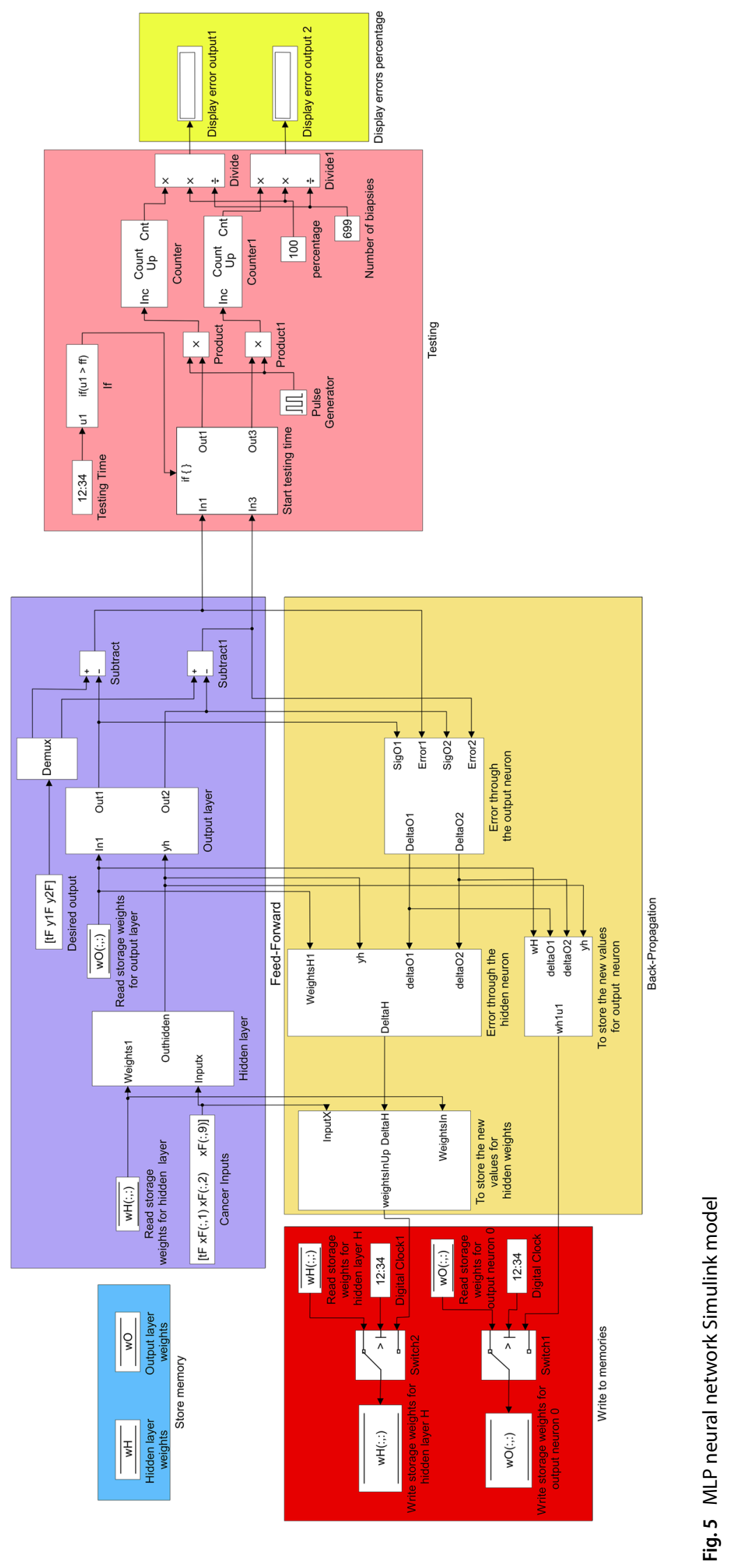




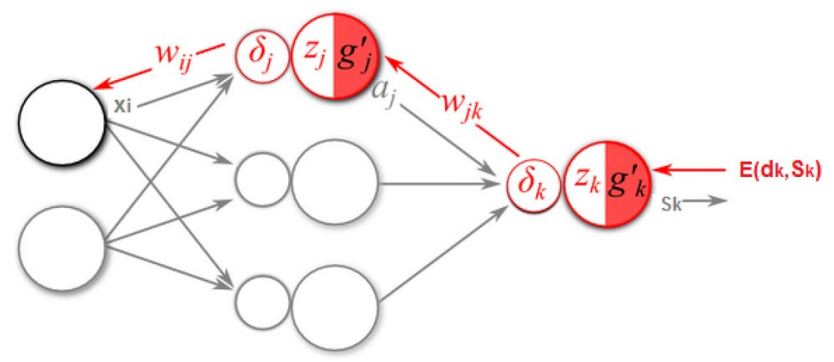

Fig. 6 Back-propagate error signals and update new values for weights

The equivalent architecture "Back-propagate error signals" block in Fig. 5 is shown in Fig. 6.

To calculate the local gradients of the output layer, $\delta_{k^{\prime}}$ used in Fig. 6, the "Back-propagate error signal O" (cf. Fig. 5) is given by Eq. 5, and is then updated with the newest weight values through the "To store the new values for output neuron" block based in the Eq. 6 (cf. Fig. 6).

$\delta_{k}=g_{k}^{\prime}\left(z_{k}\right) E\left(d_{k}, S_{k}\right) \quad \mathrm{k}=1,2$

$w_{j k}=w_{j k}+\eta a_{j} \delta_{k} \quad \mathrm{j}=1,2 \ldots 10$ and $\mathrm{k}=1,2$

To calculate the local gradients of the hidden layer, $\delta_{k^{\prime}}$ used in Fig. 6, the "Back-propagate error signal $\mathrm{H}^{\prime}$ is given by Eq. 7 , and is then updated with the newest weight values through the "To store the new values for hidden weights" block based in the Eq. 8 (cf. Fig. 6).

$\delta_{j}=g_{j}^{\prime}\left(z_{j}\right) \sum_{k=1}^{2} \delta_{k} w_{j k} \quad \mathrm{j}=1,2 \ldots 10 \mathrm{k}=1,2$

$w_{i j}=w_{i j}+\eta a_{i} \delta_{j} \quad \mathrm{i}=1,2 \ldots 9$ and $\mathrm{j}=1,2 \ldots 10$

\section{Simulation and results}

\subsection{Signals generator}

Design and analysis of analog implementation are the main objectives of this work, but before opening the model, a small program written in MATLAB generate signals and determine the times for stopping the learning and beginning the testing. This program is based on the following organization chart:

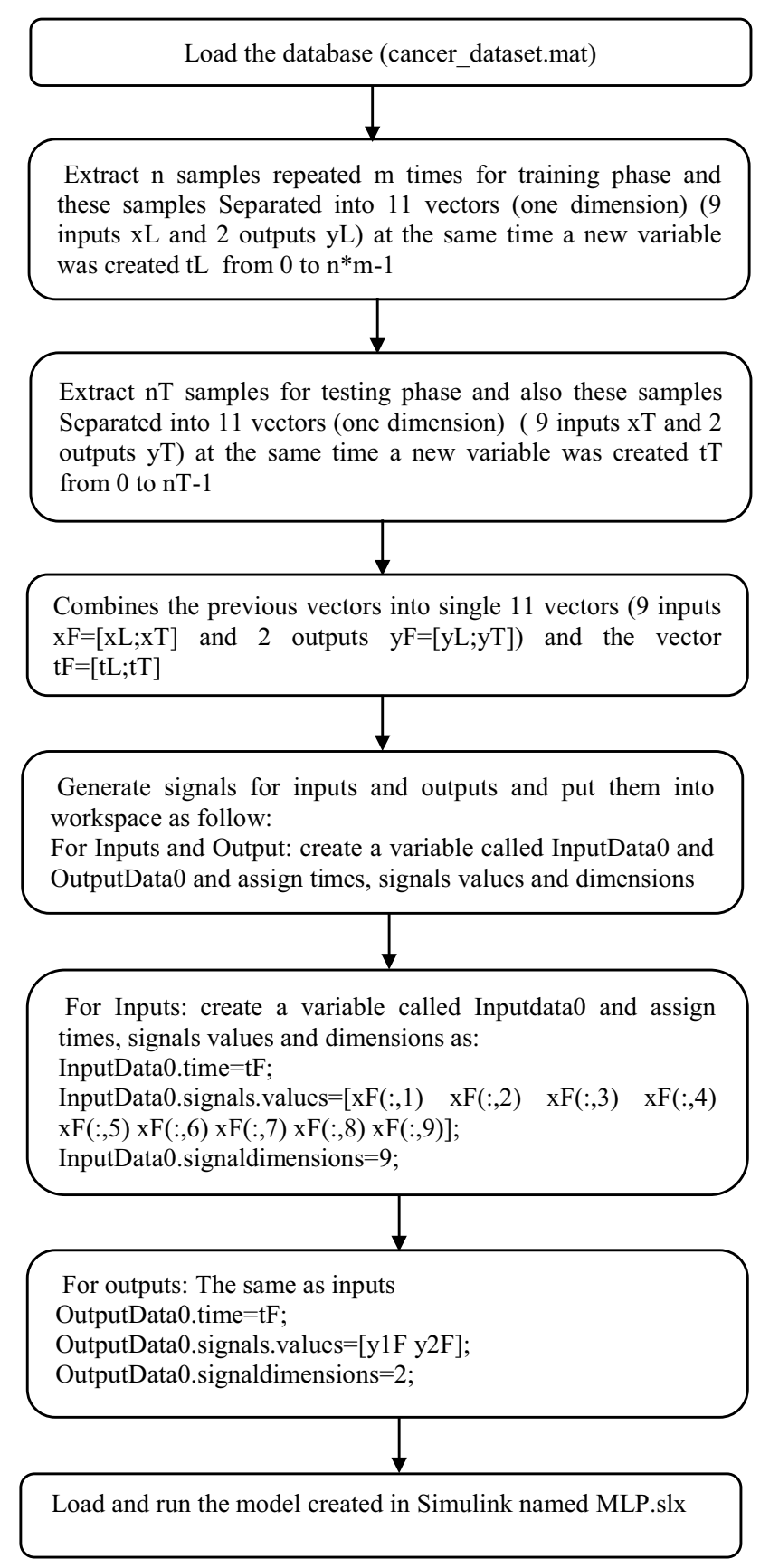

A similar organization chart was used to create signals generator and successfully tested by using the Microcontroller PIC18F2680 through software PIC C and Proteus 4.8 SF0 Pro [26], the unit time can be $0.1 \mu \mathrm{S}, 1 \mu \mathrm{S}, \ldots 1 \mathrm{~s}$ depending on the application used. 


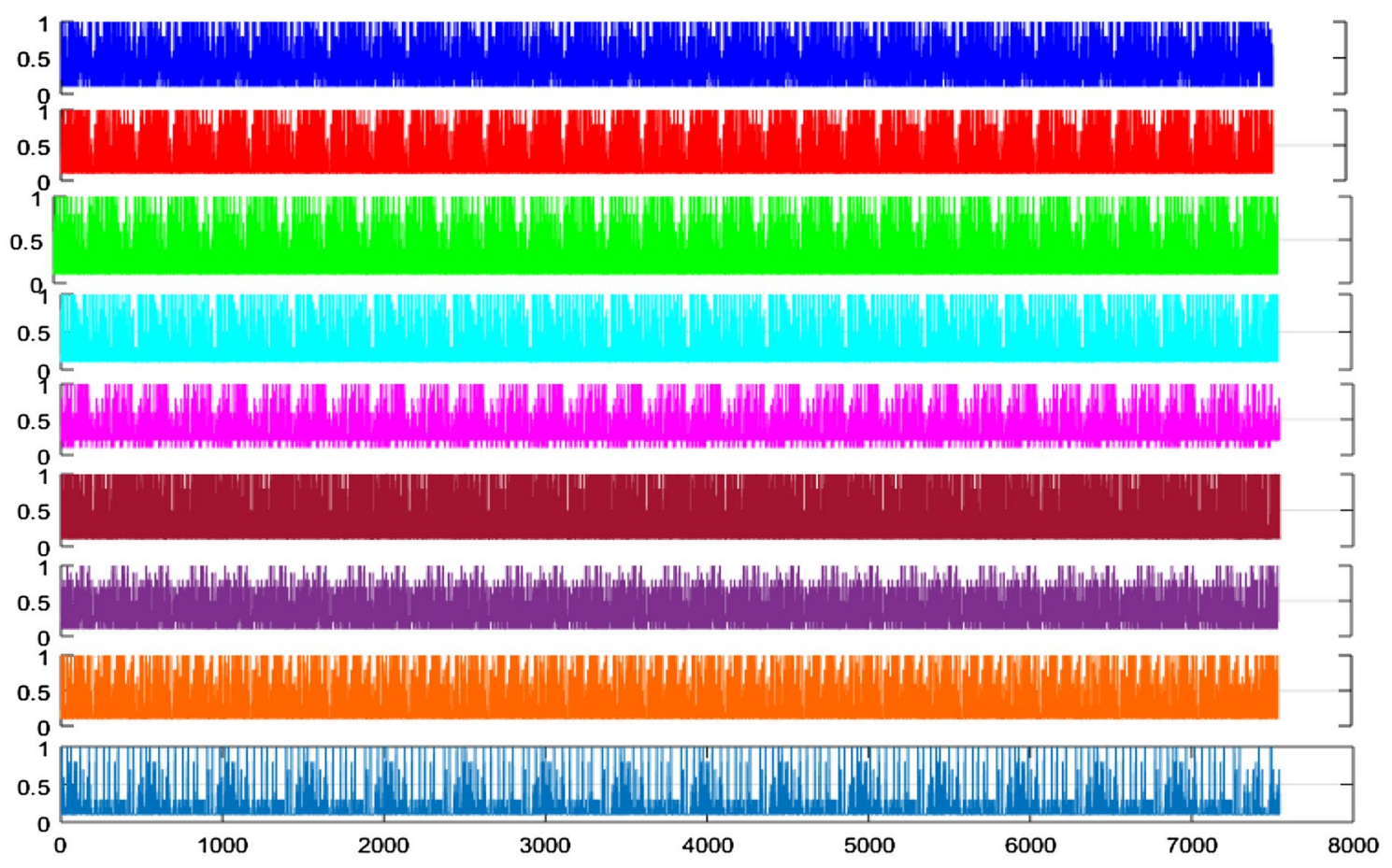

Fig. 7 Nine attributes signals

The duration time for learning phase and testing phase was calculated during running the small program that launch the model and it's equal respectively to $t L=m^{*} n\left[0\right.$ to $\left.m^{*} n-1\right]$ and $t T=\left[0+m^{*} n\right.$ to $\left.\mathrm{m}^{*} \mathrm{n}+\mathrm{tT}-1\right]$.

Two switches block determine the end of the learning phases and the beginning of the testing phase, through the variable $t L$, for example when not achieved this value during running the model, we still in learning phase.

During each unit time and during the learning phase the first switch lets the model read the weights (memories block) from the hidden layer during the first half of unit time and write the updated weights during the second half and at the end of the learning time. At the beginning of the testing phase, this switch lets the model read only from the memories block (weights in Hidden layer).

The same procedure for the second switch for the Output layer.

\subsection{Artificial neural network signals}

Before running the design shown in Fig. 5, we must use the following variables as [18]:

- Number of sample NS $=489$

- Number of epochs $=14$

- Learning rate $=0.25$.
The needed times for learning and testing phases in our model are equal to $6845 \mu$ s (i.e. $489 * 14 \mu$ s) and $7545 \mu$ s (i.e. $6845+699 \mu$ s) respectively.

Figure 7 below shows the nine attributes signals of 699 biopsies as Cancerlnputs. These values, initially between 1 and 10 in the database, are converted first into 0.1 to 1 for Matlab Simulink and into $10 \mathrm{mV}$ to $100 \mathrm{mV}$ for the analog implementation.

After executing the structure (see Fig. 5), we have the following results:

- The desired output curves for the first category (benign) and the neuron output in Fig. 8

- The desired output curves for the second category (malignant) and the neuron output in Fig. 9

- The updated weight values curve in hidden layer in Fig. 10

- The updated weight values curve in output layer in Fig. 11

Figures 8 and 9 are certainly difficult to interpret as is, but serve only as illustration. To facilitate the analysis of these curves, we have added a comparison block, presented in the next section (cf. Fig. 12). Much more interesting, Figs. 10 and 11 present the evolution of the weights in the hidden layer and that of the exit. Let us remind that these weights are 90 in the hidden layer, which is constituted by 10 neurons 

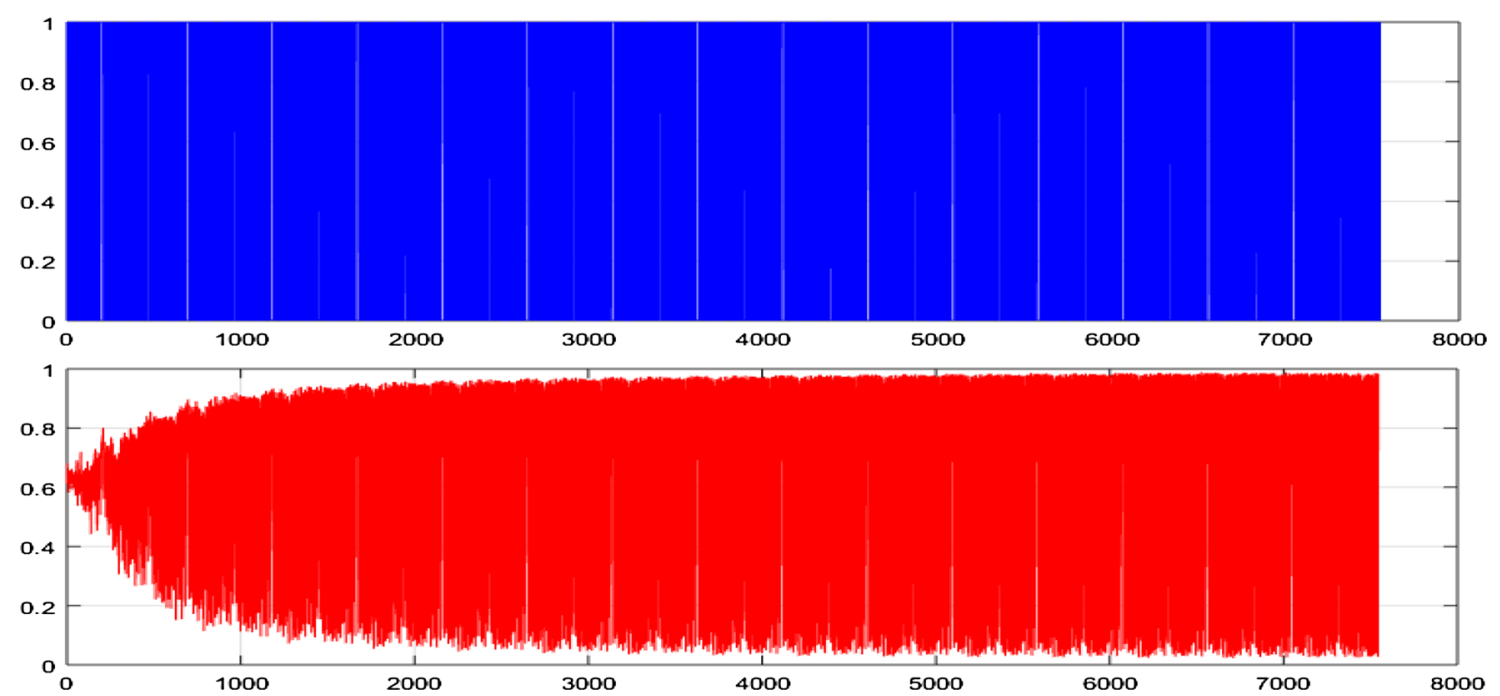

Fig. 8 First category as benign and the output produced by the neural networks
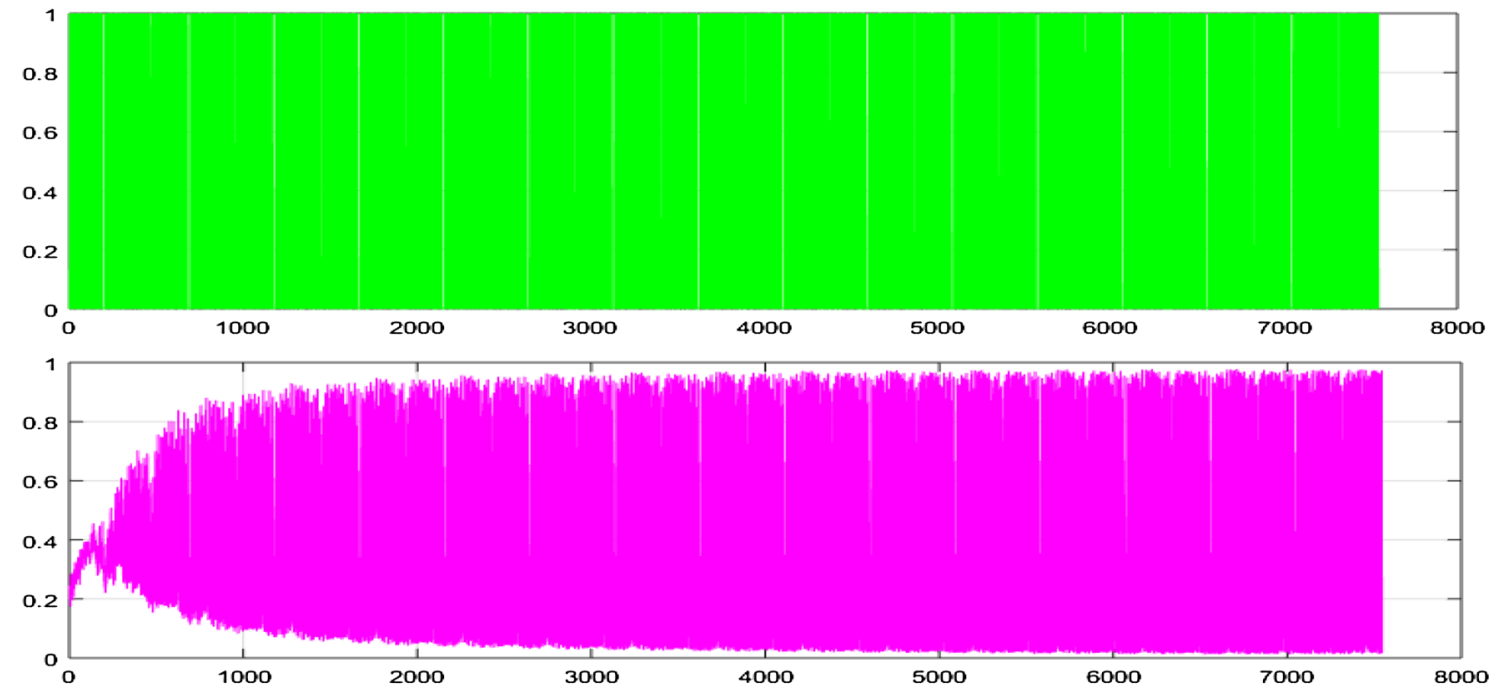

Fig. 9 Second category as malignant and the output produced by the neural networks

having each 9 inputs ( 9 attributes of every biopsy presented in Fig. 7). They are 20 in the output layer.

On Figs. 10 and 11, we can notice that at the end of $6845 \mu$ s (learning phase), the weights do not vary any more.

\subsection{Classification results}

The diagram illustrated in Fig. 12, was created to obtain the number of wrong answers produced by the neural network and the equivalent percentage shown in Fig. 13. Thanks to the structure of comparison, the number of error and thus its percentage can be calculated and offer a result easier to interpret.

As a result, we can obtain the difference between the desired output and the output created by the neural network for the first category and the second category in the upper and lower curves respectively in Fig. 13. On this figure, one 1 means the presence of a cell corresponding to its class of origin (benign for the output 1 and malignant for the output 2). For reading reasons, we printed only a part of the phase of test (upper time in $6845 \mu \mathrm{s}$ ). Although this figure is easier to interpret that the results illustrated by Figs. 8 and 9, the matrix of 


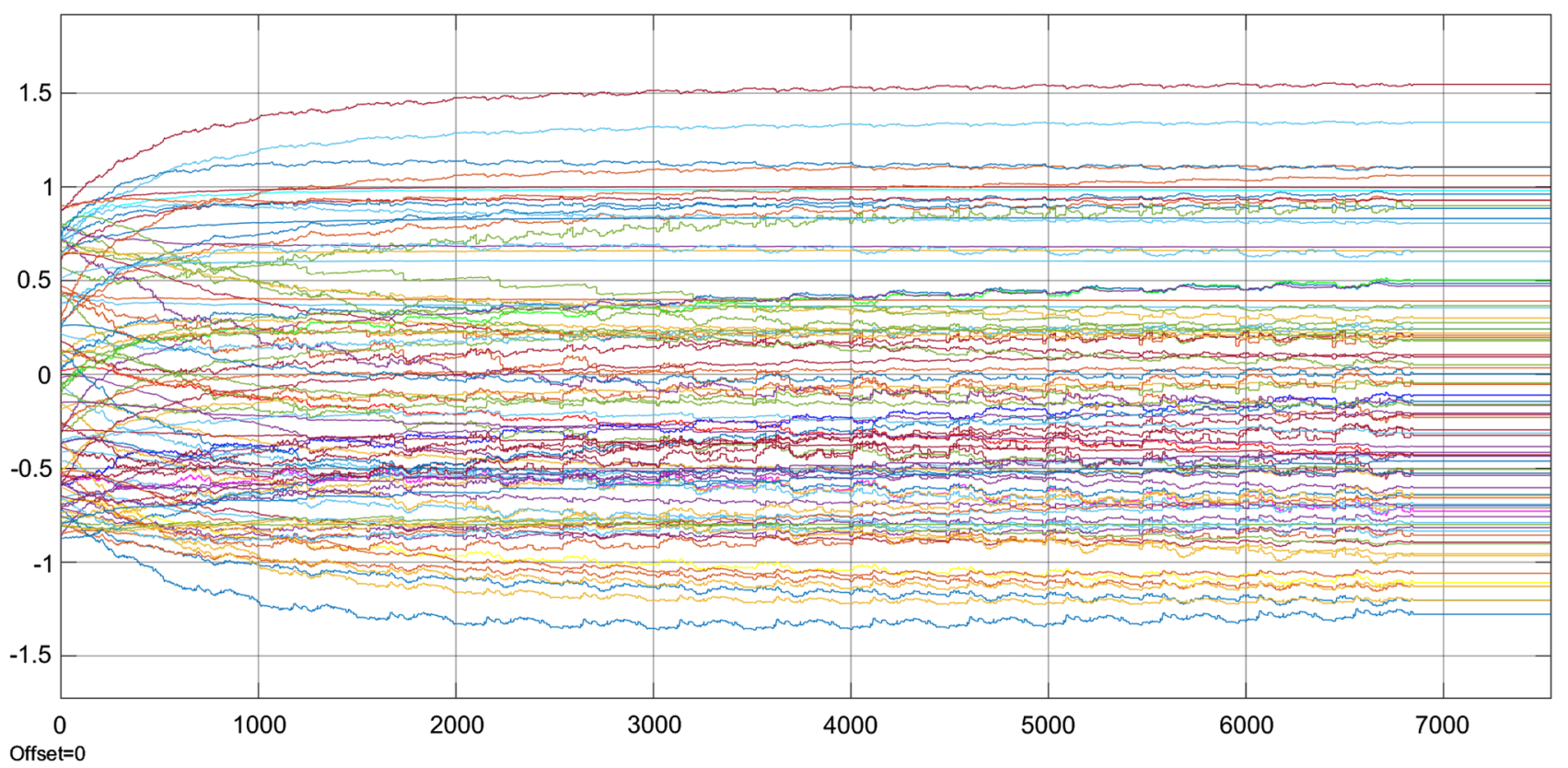

Fig. 10 Variations of weights in hidden layer

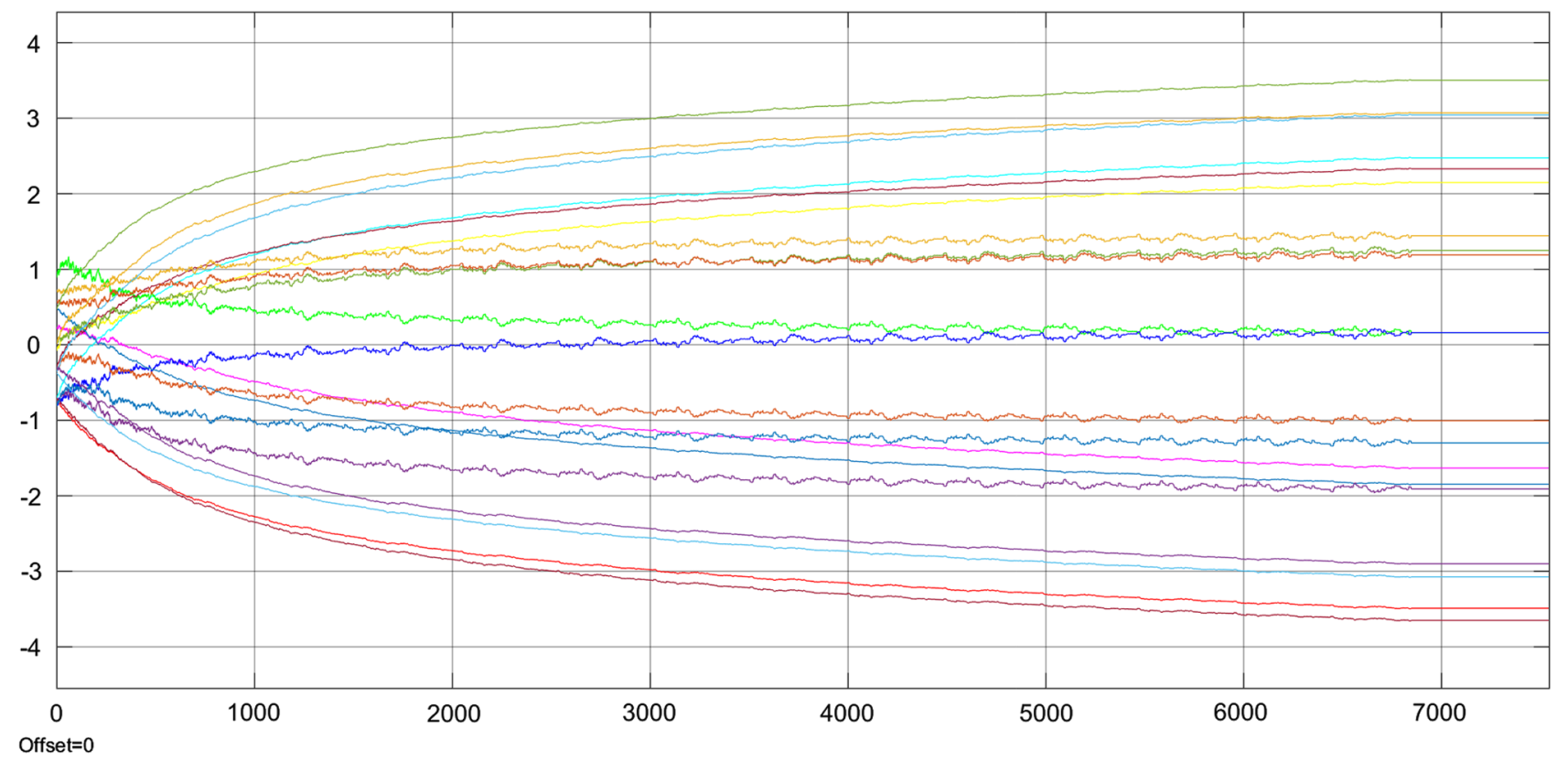

Fig. 11 Variations of weights in output layer

confusion, presented in Fig. 14, is a much simpler and more visual tool to analyse the quality of the artificial neural network.

This final result giving a total error of $2.6 \%$ was obtained with ideal blocks: multipliers, logsigmoid function and its derivative as activation function. This result serves as a reference for the following studies.

\section{Non-idealities implementation}

\subsection{Introduction}

In order to determine the influence on the non-idealities of these building blocks on the quality of the classification, we have to take into account their limitations and 


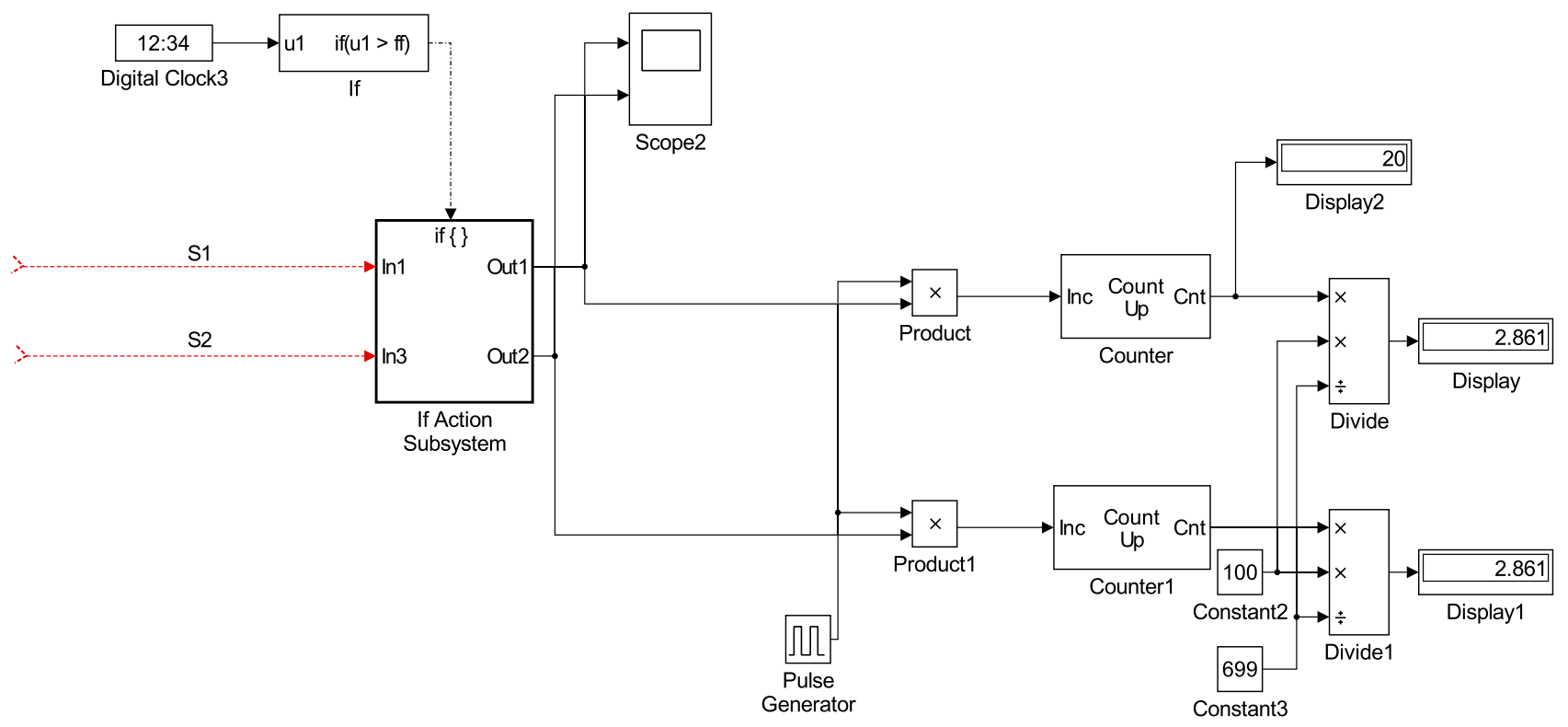

Fig. 12 Calculation of wrong answers of neural networks

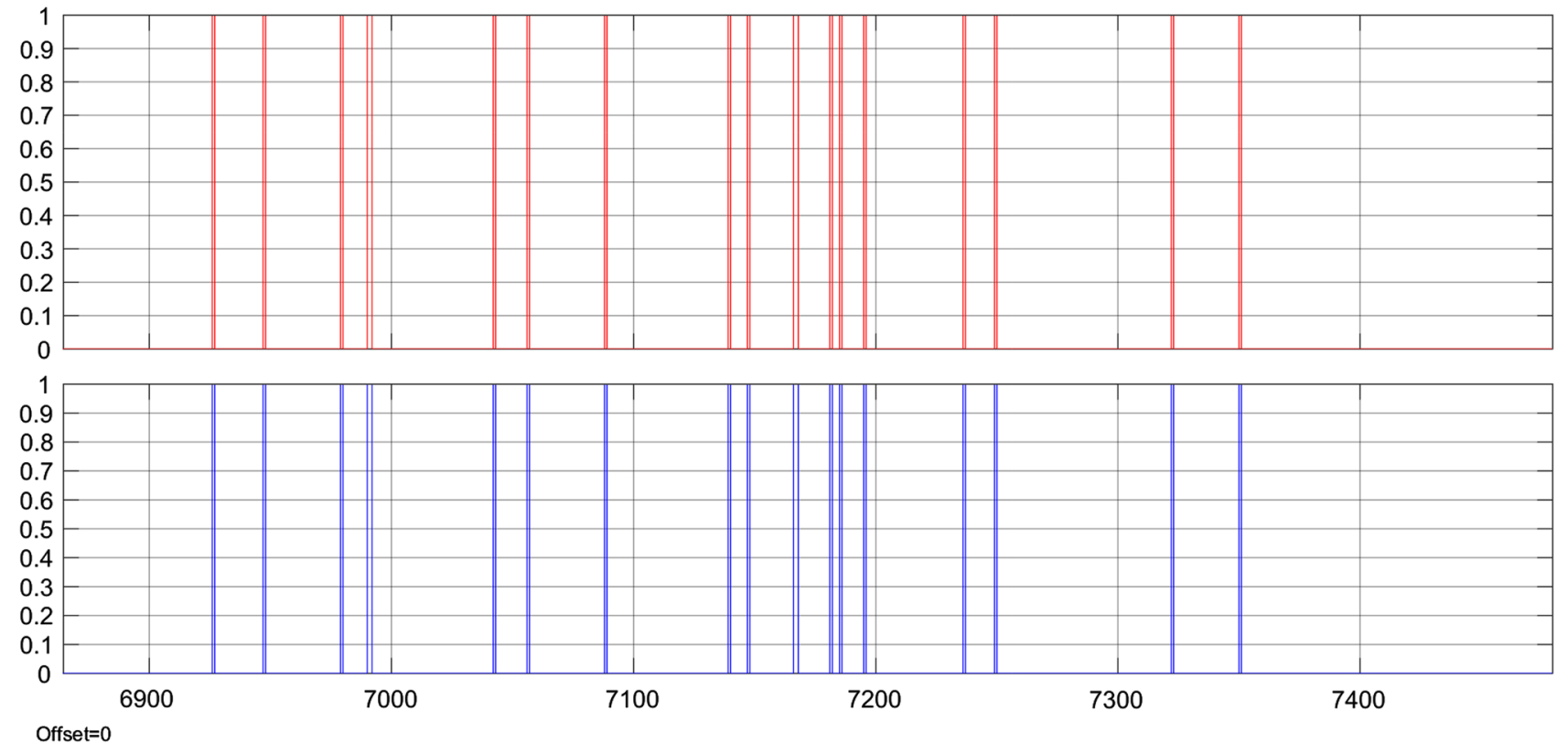

Fig. 13 Difference between the desired output and the output created by the neural network

drawbacks. This study will allow us to determine some specifications of these circuits.

\subsection{Creating a non-ideal multiplier block}

Two characteristics of the multiplier will be extracted from this study: dynamics and noise. The dynamics will be given by the extreme values of the weights (inputs) and the noise by the influence of their accuracy.

We add a random error to the multiplier block to obtain the influence of the non-ideal multiplier block on the number of wrong answers of our neural network. Figure 15 shows the diagram of this non-ideal multiplier. 


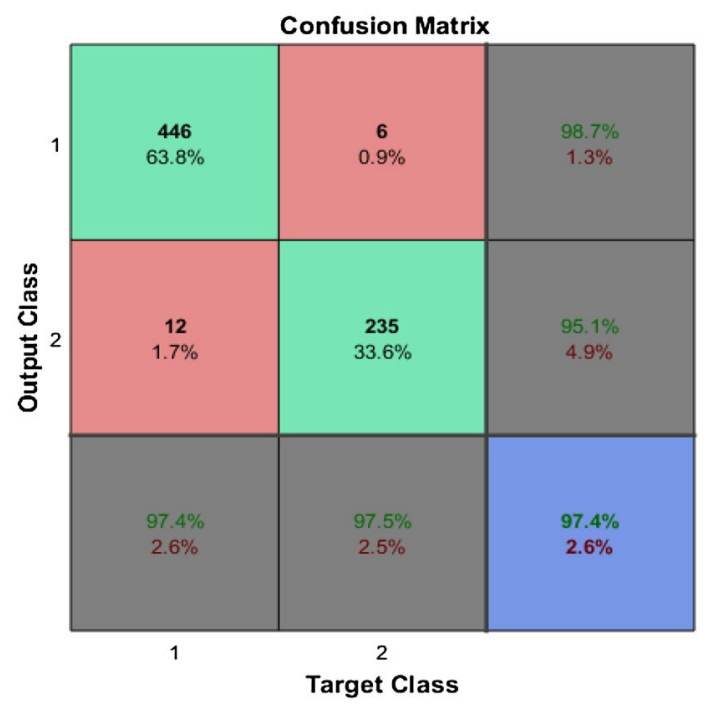

Fig. 14 Matrix of confusion with ideal building blocks

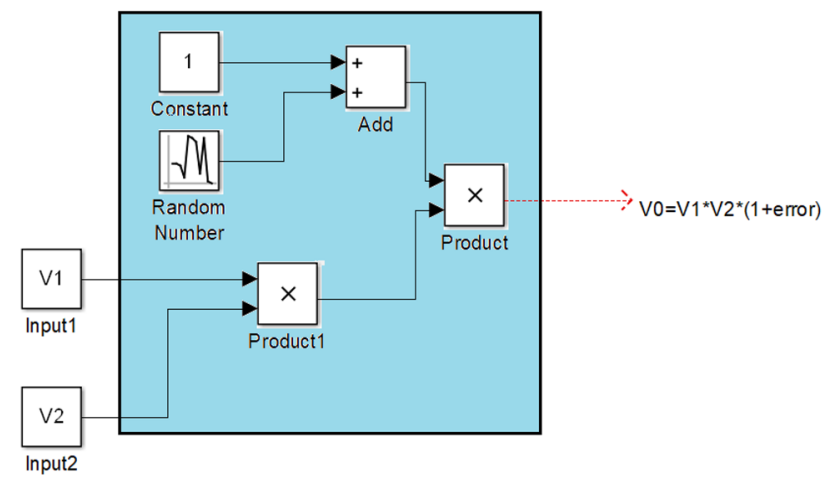

Fig. 15 Non-ideal multiplier

For example, if we choose $\mathrm{V} 1=3$ and $\mathrm{V} 2=2$, we obtain the result of V0 presented in Fig. 15 for "noisy" multiplier. The ideal value has to be equal to 6 , which is the mean value of the signal presented in Fig. 16. The aim of the following study is to determine the maximum amplitude of this random generator (i.e. the maximum input noise of the multiplier) which offers a suitable error of the classification.

By replacing the ideal multiplier block with another noisy block in the Simulink model, we create an error in the multiplier block and in the derivative function at the same time. In fact, the activation function $f(x)$ is the logsigmoid and its derivative is equal to $f(x)^{*}(1-f(x))$. For this reason, we changed at the same time the multiplier and the derivative function.

We made a series of simulations where the maximal value of the "noise" increases in relative value compared with the inputs of the multiplier. The results are summarized in the Table 4 below. The first line, with $0 \%$ of error, corresponds to the ideal case the results of which were presented previously, with a total error of $2.6 \%$.

The variables er 1 and er 2 (the first 2 columns) represent the percentages of error range between two values added to the multiplier block. The next two columns provide the total number of errors during the classification on the 699 biopsies and the corresponding percentage respectively. So, 18 total errors (cf. first line with er $1=\mathrm{er} 2=0 \%, 12$ errors for the benign class and 6 errors for the malignant class as shown in the confusion matrix, cf. Fig. 14) give a percentage error of $18 / 699=2.6 \%$. $\mathrm{wH}$ and $\mathrm{wO}$ represent the extreme values (minimum and maximum) of the weights for hidden and output layers.

These results shown that an error lower than $10 \%$ on the input of the multiplier, and so on the value of the weights, does not modify the quality of the classification; $20 \%$ with a final error of $3.3 \%$ (compared with $2.6 \%$ ) and $50 \%$ (with an error of $6.2 \%$ ) can also suit our application. Moreover, the worst case gives a dynamic (extreme values) of $[-3.8 ; 3.5]$. If we consider a scale of $5 \mathrm{mV}$ for a weight of 0.01 , a weight of 3.8 will be corresponding to a voltage of $760 \mathrm{mV}$. A final input dynamic of the multiplier, equals to $\pm 800 \mathrm{mV}$, will be enough and easily to achieve with the HCMOS9A $130 \mathrm{~nm}$ technology and a power supply of $\pm 900 \mathrm{mV}$ [12]. Also, a maximum input noise of the multiplier of $80 \mathrm{mV}(10 \%$ of error) is not a difficult constraint.

\subsection{Create a non-ideal log-sigmoid block}

By using the same previous approach, we add an error to the real log-sigmoid block presented in Fig. 17 below.

By changing the ideal logsigmoid block with another non-ideal logsigmoid block, we obtain the results presented in Table 2.

As we see in Table 5, the maximum acceptable error varies between -20 and $20 \%$ to keep the number of errors in the classification at lower value, so the activation function is very sensitive to the result of neural network.

The final step is to change the real multiplier, activation function and its derivative by other non-ideal blocks at the same time; this produces the results presented in Tables 6 and 7. Table 3 shows that the maximum acceptable range for the multiplier block is from -50 to $50 \%$ when the error range for the log-sigmoid block varies between -10 and $10 \%$.

Table 7 shows that the maximum acceptable range for the multiplier block is from -20 to $20 \%$ when the error range for the logsigmoid block varies between -20 and $20 \%$. 


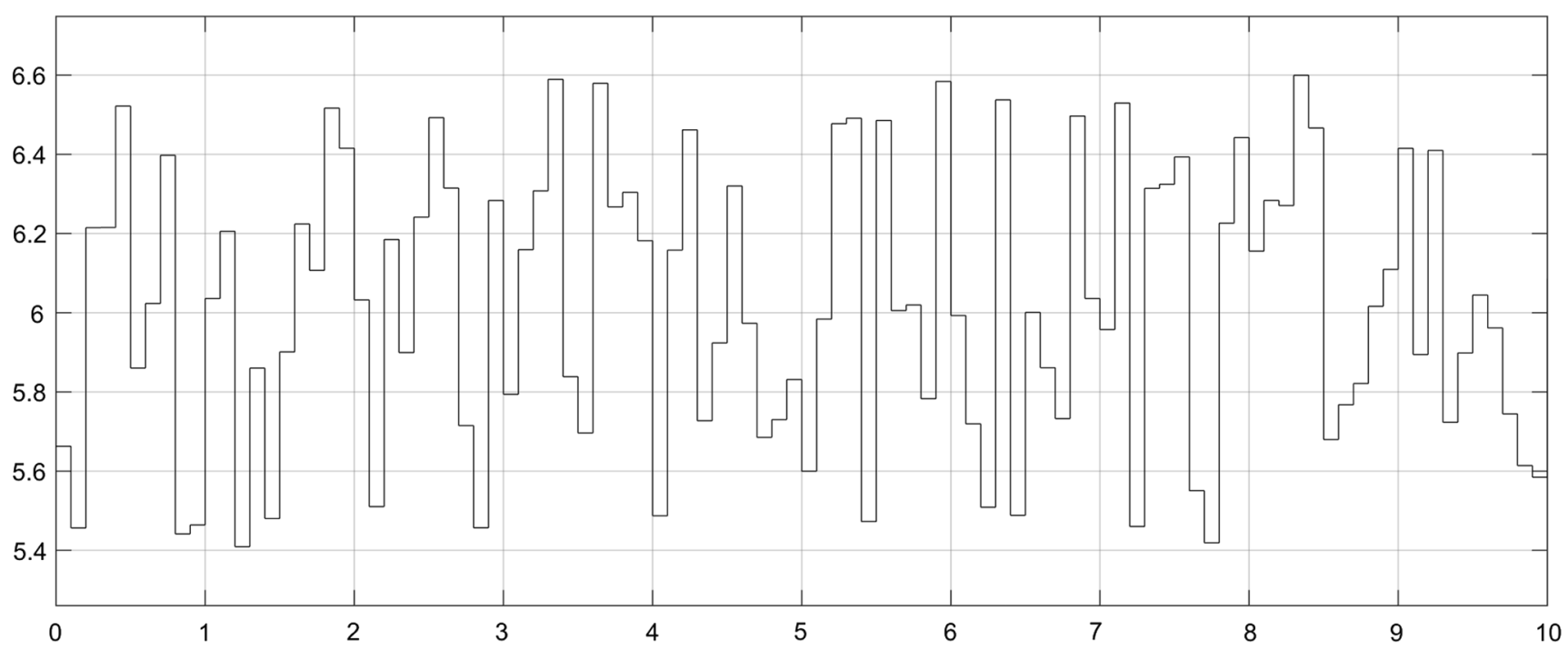

Offset $=0$

Fig. 16 Results of multiplication of 3 and 2 with the noisy multiplier

Table 4 The non-ideal multiplier and non-ideal derivative function blocks

\begin{tabular}{rclcll}
\hline er1 (\%) & er2 (\%) & Nb errors & \% Error & Extreme values: $w H$ & Extreme values: $w O$ \\
\hline 0 & 0 & 18 & 2.6 & $-1.37 \rightarrow 1.17$ & $-3.54 \rightarrow 3.48$ \\
-10 & 10 & 19 & 2.7 & $-1.6 \rightarrow 1.4$ & $-3.8 \rightarrow 3.5$ \\
-20 & 20 & 23 & 3.3 & $-1.7 \rightarrow 1.2$ & $-3.2 \rightarrow 3.8$ \\
-30 & 30 & 26 & 3.7 & $-1.7 \rightarrow 1.2$ & $-3 \rightarrow 3$ \\
-40 & 40 & 31 & 4.3 & $-1.7 \rightarrow 1.5$ & $-3 \rightarrow 3.2$ \\
-50 & 50 & 43 & 6.2 & $-1.7 \rightarrow 1.2$ & $-3 \rightarrow 2.8$ \\
-60 & 60 & 68 & 9.7 & $-1.7 \rightarrow 1.3$ & $-3 \rightarrow 2.8$ \\
-70 & 70 & 77 & 11.0 & $-1.8 \rightarrow 1.6$ & $-2.8 \rightarrow 3$ \\
-80 & 80 & 86 & 12.3 & $-2.5 \rightarrow 1.8$ & $-2.5 \rightarrow 3.1$ \\
-90 & 90 & 92 & 13.6 & $-2.4 \rightarrow 1.8$ & $-2.7 \rightarrow 2.5$ \\
-100 & 100 & 98 & 14.0 & $-2.8 \rightarrow 1.8$ & $-2.7 \rightarrow 2.5$ \\
\hline
\end{tabular}

\section{Results analysis}

In analog CMOS circuits we can't find, create or fabricate an ideal multiplier or an ideal activation function. For this reason, a non-ideal multiplier and a non-ideal log-sigmoid were designed to verify the acceptable range to be used in the analog CMOS circuits.

$\mathrm{wH}$ and wO represent respectively the weights in Hidden and Output layers and these weights change during the learning phase. In software world no limits for range contrary to CMOS world where this range varies from circuit to another.

From Table 4 we can deduce that in CMOS world for example: 
Table 5 The non-ideal logsigmoid block

\begin{tabular}{llcccl}
\hline er3 $(\%)$ & er4 $(\%)$ & Nb errors & \% Error & Extreme values: $w H$ & Extreme values: wO \\
\hline-10 & 10 & 24 & 3.4 & $-1.6 \rightarrow 1.4$ & $-3.2 \rightarrow 3.8$ \\
-20 & 20 & 49 & 7.0 & $-1.7 \rightarrow 1.2$ & $-4 \rightarrow 6.2$ \\
-25 & 25 & 241 & 34.5 & $-1.7 \rightarrow 1.2$ & $-4 \rightarrow 16$ \\
\hline
\end{tabular}

Table 6 Error changes between $-10 \%$ and $10 \%$ for logsigmoid block
Table 7 Error changes between $-20 \%$ and $20 \%$ for logsigmoid block

\begin{tabular}{llllllll}
\hline er1 (\%) & er2 (\%) & er3 (\%) & er4 (\%) & Nb errors & \% Error & Extreme wH & Extreme wO \\
\hline-10 & 10 & -10 & 10 & 22 & 3.1 & $-1.6 \rightarrow 1.2$ & $-3.9 \rightarrow 4.1$ \\
-20 & 20 & -10 & 10 & 24 & 3.4 & $-1.6 \rightarrow 1.3$ & $-3.6 \rightarrow 3.6$ \\
-30 & 30 & -10 & 10 & 28 & 4.0 & $-1.4 \rightarrow 1.5$ & $-3 \rightarrow 3$ \\
-40 & 40 & -10 & 10 & 30 & 4.3 & $-1.6 \rightarrow 1.0$ & $-4 \rightarrow 3.4$ \\
-50 & 50 & -10 & 10 & 38 & 5.4 & $-1.7 \rightarrow 1.5$ & $-3.2 \rightarrow 3.4$ \\
-60 & 60 & -10 & 10 & 241 & 34.5 & $-5.2 \rightarrow 6.5$ & $-6 \rightarrow 48$ \\
\hline
\end{tabular}

\begin{tabular}{llllcccc}
\hline er1 (\%) & er2 (\%) & er3 (\%)1 & er4 (\%) & Nb errors & \% Error & Extreme wH & Extreme wO \\
\hline-10 & 10 & -20 & 20 & 48 & 6.9 & $-2.2 \rightarrow 1.2$ & $-4 \rightarrow 6.2$ \\
-20 & 20 & -20 & 20 & 241 & 34.5 & $-2.1 \rightarrow 2.3$ & $-3.8 \rightarrow 11$ \\
\hline
\end{tabular}

Case 2 (row 2) when we have an error between -10 and $+10 \%$ applied to the multiplier the extreme values for $\mathrm{wH}$ and $\mathrm{wO}$ respectively will be $-160 \mathrm{mV} \rightarrow 140 \mathrm{mV}$ and $-380 \mathrm{mV} \rightarrow 350 \mathrm{mV}$.

The same for the activation function for example from Table 5:

Case 2 (row 2) when we have an error between -20 and $+20 \%$ applied to the log-sigmoid the extreme values for $\mathrm{wH}$ and $\mathrm{wO}$ respectively will be $-170 \mathrm{mV} \rightarrow 120 \mathrm{mV}$ and $-400 \mathrm{mV} \rightarrow 620 \mathrm{mV}$.

When we choose the blocks together and from Table 7:

Case 1(row 1) when we have an error between -10 and $+10 \%$ applied to the multiplier and when we have an error between -20 and $+20 \%$ applied to the log-sigmoid, the extreme values for $\mathrm{wH}$ and $\mathrm{wO}$ respectively will be $-220 \mathrm{mV} \rightarrow 120 \mathrm{mV}$ and $-400 \mathrm{mV} \rightarrow 620 \mathrm{mV}$.

These results show that $\mathrm{a} \pm 800 \mathrm{mV}$ input dynamics of the multiplier is sufficient, since it gives a very high sensitivity of $5 \mathrm{mV}$ for a 0.01 accuracy of the coefficients (i.e. weights of the neurons), well beyond than we need. Likewise, an error of $10 \%$ (or 20\%) on these inputs corresponds to an input noise of the multiplier of $80 \mathrm{mV}$ (or $160 \mathrm{mV}$ respectively). These specifications will be very easy to perform with the chosen $130 \mathrm{~nm}$ CMOS HCMOS9A technology and with a power supply of the electronics of $\pm 900 \mathrm{mV}$ [12].

In addition, to simplify the final realization of the artificial neural network, we chose a current output of the multipliers. Thus, the addition of multipliers' outputs is realized by using simply the Kirchhoff law for feeding the activation function. The constraints of this latter are not in voltage but in current; its dynamics will therefore depend on the realization of the multiplier with however a noise constraint which not exceed $10 \%$ of the value.

\section{Conclusion}

After creation and simulation for different errors on the non-ideal blocks for the multiplier, activation function and its derivative, this procedure gives us information about the acceptable error when we want to create a neural network by using the analog CMOS circuits.

From this study, we conclude that the activation function log-sigmoid block is very sensitive to the wrong answer for the classification of breast cancer detection.

In future projects, we will try to build the activation function log-sigmoid by using the analog CMOS circuit, taking into consideration the maximum acceptable range for this block.

Acknowledgements The authors would like to acknowledge the support of the Lebanese International University, and Polytech'Lab,.

Funding This study was funded by the Lebanese International University, and Polytech'Lab.

\section{Compliance with ethical standards}

Conflict of interest The authors declare that they have no conflict of interest. 


\section{References}

1. Aličković $E$, Subasi $A$ (2017) Breast cancer diagnosis using $G A$ feature selection and Rotation Forest. Neural Comput Appl 28(4):753-763

2. Aličković E, Subasi A (2011) Data mining techniques for medical data classification. In: The international Arab conference on information technology (ACIT)

3. Abbas HA (2001) An evolutionary artificial neural network approach for breast cancer diagnosis. Artif Intell Med 25:265-281

4. Hassan MR, Hossain MM, Begg RK, Ramamohanarao K, Morsi Y (2010) Breast-cancer identification using HMM-fuzzy approach. Comput Biol Med 40:240-251

5. Alickovic E, Subasi A (2019) Normalized neural networks for breast cancer classification. In: International conference on medical and biological engineering. Springer, Cham, pp 519-524

6. Liu J, Liang D (2005) A survey of FPGA-based hardware implementation of ANNs. In: 2005 International conference on neural networks and brain, vol 2. IEEE, pp 915-918

7. Alçın M, Pehlivan I, Koyuncu İ (2016) Hardware design and implementation of a novel ANN-based chaotic generator in FPGA. Optik 127(13):5500-5505

8. Sahin S, Becerikli Y, Yazici S (2006) Neural network implementation in hardware using FPGAs. In: International conference on neural information processing. Springer, Berlin, Heidelberg, pp 1105-1112

9. Dias FM, Antunes A, Mota AM (2004) Artificial neural networks: a review of commercial hardware. Eng Appl Artif Intell 17(8):945-952

10. Valle M, Caviglia DD, Bisio GM (1994) Back propagation learning algorithm for analog VLSI imple-mentation. In: Delgado JG, Moore WR (eds) VLSI for neural network and artificial intelligence. Plenum Press, New York, pp 35-44

11. Hirai Y (1993) Recent VLSI neural networks in Japan. JVLSI Signal Process 6(1):7-18

12. Jouni H, Harb A, Jacquemod G, Leduc $Y$ (2017) Wide range analog CMOS multiplier for neural network application. In: EEETEM2017, Beirut
13. Kenzhina M, Dolzhikova I (2018) Analysis of hyperbolic tangent passive resistive neuron with CMOS-memristor circuit. In: Second international conference on computing and network communications (CoCoNet'18)

14. Heidari M, Shamsi H (2019) Analog programmable neuron and case study on VLSI implementation of multi-layer perceptron (MLP). Microelectron J 84:36-47

15. Jayadeva Dr, Deb A, Chandra S (2002) Algorithm for building a neural network for function approximation. IEEE Proc Circuits Devices Syst 149:301-307

16. da Silva IN, Hernane Spatti D, Andrade Flauzino R, Liboni LHB, dos Reis Alves SF (2017) Multilayer perceptron networks. In: Artificial neural networks. Springer, Cham

17. Salama Gl, Abdelhalim MB, Zeid MA (2012) Breast cancer diagnosis on three different datasets using multi-classifiers. Int J Comput Inf Technol 01(01):2277-0764

18. Jouni H, Issa M, Harb A, Jacquemod G, Leduc Y (2016) Neural network architecture for breast cancer detection and classification. IMCET, Beirut

19. MathWorks (2016) MATLAB and statistics toolbox release $2016 a$. The MathWorks Inc, Natick, Massachusetts, United States

20. https://medlineplus.gov/magazine/issues/summer14/articles/ summer14pg20.html

21. https://www.cancer.org/cancer/breast-cancer/about/what-isbreast-cancer.htm

22. Frank A, Asuncion A (2010) UCI machine learning repository. School of Information and Computer Science, University of California, Irvine. http://archive.ics.uci.edu/ml

23. Matlab Neural Network Toolbox. http://www.mathworks.com/

24. Thanh NP, Kung YS, Chen SC, Chou HH (2016) Digital hardware implementation of a radial basis function neural network. Comput Electr Eng 53:106-121

25. Tisan A, Cirstea M (2013) SOM neural network design-A new Simulink library based approach targeting FPGA implementation. Math Comput Simul 91:134-149

26. Jouni H, Harb A, Jacquemod G, Leduc $Y$ (2017) Programmable signal generator for neural network application. ICM, Beirut

Publisher's Note Springer Nature remains neutral with regard to jurisdictional claims in published maps and institutional affiliations. 